\title{
Preditores do Desempenho Escolar ao final do Ensino Fundamental: Histórico de Reprovação, Habilidades Sociais e Apoio Social
}

\author{
Luana de Mendonça Fernandes ${ }^{1, *}$ \\ Orcid.org/0000-0002-7325-7881 \\ Vanessa Barbosa Romera Leme ${ }^{2}$ \\ Orcid.org/0000-0002-9721-0439 \\ Luciana Carla dos Santos Elias ${ }^{3}$ \\ Orcid.org/0000-0002-1623-0674 \\ Adriana Benevides Soares ${ }^{1}$ \\ Orcid.org/0000-0001-8057-6824
}

\author{
${ }^{1}$ Universidade Salgado de Oliveira, Niterói, RJ, Brasil \\ ${ }^{2}$ Universidade do Estado do Rio de Janeiro, Rio de Janeiro, RJ, Brasil \\ ${ }^{3}$ Universidade de São Paulo, Ribeirão Preto, SP, Brasil
}

\section{Resumo}

A literatura tem indicado que o desenvolvimento socioemocional dos alunos pode ser associado a seu repertório de habilidades sociais e percepção de apoio social, enquanto a experiência de reprovação parece influenciar negativamente esse desenvolvimento e o desempenho escolar ao final do Ensino Fundamental (E.F.). Diante desse contexto, o presente estudo teve por objetivo testar um modelo de predição para o desempenho escolar, tendo como variáveis independentes as habilidades sociais, a percepção de apoio social da família, professores e pares e o histórico de reprovação dos estudantes. Participaram 311 alunos do $8^{\circ}$ e $9^{\circ}$ ano do E.F., provenientes de escolas públicas do Estado do Rio de Janeiro. Os instrumentos utilizados na coleta de dados foram: Inventário de Habilidades Sociais para Adolescentes e Escala de Percepção de Apoio Social. O histórico de reprovação, as habilidades sociais e percepção de apoio social do professor predisseram o desempenho escolar dos alunos. A partir desses resultados, discute-se a importância dos recursos dos alunos e do seu contexto que poderão ser utilizados na escola através de programas de prevenção e promoção do bom desempenho escolar com os alunos, as famílias e os professores.

Palavras-chave: Habilidades sociais, percepção de apoio social, desempenho escolar, reprovação, Ensino Fundamental.

\section{Predictors of Academic Achievement at the End of Middle School: History of Repetition, Social Skills and Social Support}

\begin{abstract}
The literature has shown that the socio-emotional development of students can be associated with their repertoire of social skills and perception of social support, while the school repetition experience seems
\end{abstract}

* Endereço para correspondência: Rua Maestro José Botelho, 2, Apto 203, Vital Brasil, Niterói, RJ, Brasil 24230410. Fone: (21) 2711-9291. E-mail: luapsi@gmail.com 
to negatively influence their development and academic achievement at the end of Middle School. In this context, the present study aimed to test a prediction model for academic achievement, having social skills, perception of social support from family, teachers and peers and history of school repetition of students as independent variables. The participants were 311 at the end of Middle School $\left(8^{\text {th }}\right.$ and $9^{\text {th }}$ grade), from public schools in the state of Rio de Janeiro. The data were collectively obtained using the Social Skills Inventory for Adolescents and the Social Support Appraisal Scale. The experience of school repetition, social skills and perceived social support from teachers predicted the academic achievement of students. From these results, the importance of the resources of students and of their context is discussed. These can be used in the school through programs of prevention and promotion of good academic achievement with students, families and teachers.

Keywords: Social skills, social support, academic achievement, school repetition, middle school.

\section{Los Predictores de Rendimiento Académico al Final de la Educación Primaria: Historial de la Desaprobación, Habilidades Sociales y Apoyo Social}

\section{Resumen}

La literatura ha demostrado que el desarrollo socio-emocional de los estudiantes se puede asociar con su repertorio de habilidades sociales y la percepción de apoyo social, mientras que la experiencia de la desaprobación parece influir negativamente en este desarrollo y el rendimiento académico al final de la educación primaria. En este contexto, el presente estudio tuvo como objetivo poner a prueba un modelo de predicción de rendimiento académico, teniendo como variables independientes las habilidades sociales, la percepción de apoyo social de la familia, los maestros y los compañeros y la historial de la desaprobación de los estudiantes. 311 estudiantes participaron en el grado 8 y 9 de la educación primaria, de las escuelas públicas en el estado de Río de Janeiro. Los instrumentos utilizados en la recogida de datos con los estudiantes fueron: Inventario de Habilidades Sociales para Adolescentes y la Escala de percepción de apoyo social. La experiencia de la desaprobación, habilidades sociales y profesor de apoyo social percibido predice el rendimiento académico de los estudiantes. A partir de estos resultados, se discute la importancia de los recursos de los estudiantes y su contexto que se pueden utilizar en la escuela a través de la prevención y la promoción de programas de actuación buena escuela con estudiantes, familias y profesores.

Palabras clave: Habilidades sociales, apoyo social, rendimiento académico, desaprobación, educación primaria.

O sucesso escolar nem sempre é alcançado por todos os alunos ao longo dos ciclos escolares. Tarefas repetitivas, ausência de relação entre os conteúdos escolares e vivências dos alunos, condições de trabalho docente precárias, avaliações maçantes e rígidas têm saturado o ambiente escolar, prejudicando o processo ensino-aprendizagem (Zambon \& Rose, 2012). Tais dificuldades enfrentadas pelos estudantes ao longo do percurso escolar podem ser analisadas por meio do fenômeno do fracasso escolar que se manifesta pelos problemas na aprendizagem, problemas de comportamentos, baixo desempenho escolar, reprovações, evasões e abandonos (Dazzani, Cunha, Luttigards, Zucoloto, \& Santos, 2014; Marturano \& Elias, 2016).

Diversas pesquisas documentam há alguns anos os efeitos negativos das dificuldades escolares e o baixo desempenho escolar para o desenvolvimento socioemocional e comportamental dos alunos, como por exemplo, menor autoestima e motivação para estudar e maiores níveis de estresse (D'Abreu \& Marturano, 2010; Inglés, Martínez-González, García-Fernández, 
\& Ruiz-Esteban, 2011). Assim, o presente estudo abordará algumas variáveis, tais como as habilidades sociais, percepção de apoio social e reprovação que podem contribuir para uma trajetória escolar de sucesso ou não de estudantes nos anos finais do Ensino Fundamental (E.F). Esse período do percurso acadêmico caracteriza-se como um momento crítico na trajetória escolar dos alunos. Dados do relatório sobre Índice de Desenvolvimento da Educação Básica (Ideb, 2013) evidenciaram que um total aproximado de 473.435 estudantes que frequentavam os anos finais do E.F. abandonaram a escola durante o ano de 2013. Desse modo, parece pertinente focalizar esse período de transição escolar para buscar identificar fatores relacionados à permanência e à conclusão do E.F. e também ao ingresso no Ensino Médio.

O desempenho escolar pode ser entendido como a capacidade que os alunos têm de expressar sua aprendizagem e seu conhecimento adquirido no processo ensino-aprendizagem (Perrenoud, 2003). Esse infere nas habilidades acadêmicas dos alunos e tem caráter avaliativo na medida em que os estudantes devem demonstrar em suas respostas em testes e provas, por exemplo, o que aprenderam nas aulas. Segundo D'Abreu e Marturano (2010), o baixo desempenho escolar ocorre quando o aluno apresenta, em notas ou tarefas, um resultado abaixo do nível esperado para a sua idade, habilidade e potencial de um indivíduo.

Numa concepção que supera a culpabilização dos alunos pelo fracasso escolar, o desempenho escolar deve ser compreendido como um fenômeno multideterminado, influenciado por aspectos dos estudantes, dos seus contextos familiares e escolares, assim como por fatores socioculturais, institucionais, políticos e econômicos (Dazzani et al., 2014; Marturano \& Pizato, 2015; Moreira, Rosário, \& Santos, 2011; Wang \& Holcombe, 2010). Dentre essas variáveis, pesquisas indicam que as habilidades sociais e a percepção de apoio social dos alunos podem ser compreendidas como fatores de proteção frente as adversidades (como a reprovação), afetando positivamente o desempenho escolar (Dias, Oliveira, Moreira, \& Rocha, 2015; Glozah \& Peva- lin, 2014; Lopes, Del Prette, \& Del Prette, 2013; Loureiro, Ferreira, \& Santos, 2013; Mackinnon, 2012; Nunes, Pontes, Silva, \& Dell'Aglio, 2014).

No que tange a reprovação, segundo o censo escolar realizado pelo Instituto Nacional de Estudos e Pesquisas Educacionais Anísio Teixeira (Inep), em 2015, as taxas de reprovação são significativamente maiores nos anos finais do E.F., o que contribuem para a distorção idade-série e, consequentemente, para o abandono escolar. Em 2015, 170.440 alunos evadiram no primeiro ano do E.F., enquanto que no último ano esse número subiu para 450.317 alunos (Inep, 2015).

O sistema de ensino brasileiro avalia seus alunos de várias maneiras, sendo que alguns Estados adotam o sistema de progressão continuada que consiste em uma avaliação por ciclos. $\mathrm{Ou}$ seja, os alunos para poderem avançar para um novo ciclo precisam adquirir competências e habilidades em um ciclo (por exemplo, do $1^{\mathrm{a}}$ ao $3^{\circ}$ ano, do $4^{\circ}$ ao $6^{\circ}$ ano e do $7^{\circ}$ ao $9^{\circ}$ ano) que é mais longo que um ano ou uma série. Nesse modo de avaliação, os alunos não reprovam de ano $\mathrm{e}$ podem fazer recuperação por meio de aulas de reforço para avançar para um novo ciclo (Jacomini, 2004). O Estado do Rio de Janeiro, aonde foi realizada a pesquisa, o regime de avaliação é o seriado em que os alunos podem reprovar por série/ano escolar e não por ciclo. Assim, os alunos são avaliados ano a ano por meio de provas, participação em sala de aula e relacionamento interpessoal e precisam obter uma média mínima para "passar de ano" (Alves, 2013).

A literatura tem apontado que alunos que já reprovaram apresentam sentimento de fracasso, se sentem menos motivados e com menor perspectiva quanto à continuidade dos estudos e ao futuro (Faissol \& Bastos, 2014; Souza, Ponczec, Oliva, \& Tavares, 2012). Dificuldades escolares, entre elas a reprovação, favorecem o surgimento de problemas comportamentais e emocionais, podendo produzir descrença nos professores, nos pais e nos próprios alunos quanto a sua capacidade de aprender (Ingul, Klockner, Silverman, \& Nordahl, 2012). Ortigão e Aguiar (2013) evidenciaram que os alunos com baixo desempenho escolar, geralmente, são aqueles que já vivencia- 
ram um ou mais episódios de reprovação escolar. Na mesma direção, pesquisas (Alves, Ortigão, \& Franco, 2007; Bandeira, Rocha, Pires, Del Prette, \& Del Prette, 2006; Jacomini, 2009) mostraram que os alunos que passaram por repetência podem repetir a situação de fracasso escolar nos próximos anos de vida acadêmica. Nunes et al. (2014) realizaram um estudo com 610 jovens do Ensino Fundamental e Médio, com o objetivo de investigar relações entre reprovação escolar, percepções quanto a escola e expectativa de futuro. Os resultados mostraram que quase $50 \%$ dos alunos tinham histórico de reprovação escolar e, desses, 3,9\% tinham sido expulsos da escola. Os resultados também indicaram que quanto maior o índice de reprovação, menor é a expectativa de entrar na universidade, sendo, portanto, a reprovação escolar um fator de risco psicossocial. Contudo, se as reprovações afetam negativamente o desempenho escolar, recursos dos alunos e dos seus contextos, tais como as habilidades sociais e o apoio social da família, professores e pares podem funcionar como fator de proteção.

As habilidades sociais podem ser compreendidas como comportamentos de um indivíduo, aceitáveis socialmente dentro de um contexto, de uma cultura e de um tempo histórico que favorecem relacionamentos interpessoais pautados no respeito aos direitos humanos (Del Prette \& Del Prette, 2010). As habilidades sociais dos alunos, como por exemplo, pedir ajuda, oferecer apoio, iniciar e manter conversação, recusar pedidos sem prejudicar a relação entre outras tem sido recorrentemente associado positivamente com o bom desempenho escolar (Hall \& DiPerna, 2016; Lopes et al., 2013). Marturano e Pizato (2015) ao testar um modelo preditivo encontraram que as habilidades sociais com alunos do $3^{\circ}$ ano do E.F. explicaram cerca de $40 \%$ da variabilidade do desempenho escolar no $5^{\circ}$ ano.

Estudos sugerem que alunos com déficits em habilidades sociais e controle emocional insuficiente demonstraram maior dificuldade em socializar com colegas e professores, podendo vivenciar insucesso acadêmico e, posteriormente, uma saída prematura da escola (Beau- champ \& Anderson, 2010; Elias \& Marturano, 2014; Feitosa, Del Prette, \& Del Prette, 2012). De modo geral, quanto maior for o repertório de habilidades sociais dos alunos melhor o desempenho escolar (Caemmerer \& Keith, 2015; Cia \& Costa, 2012; Marturano \& Pizato, 2015; Womack, Marchant, \& Borders, 2011).

Com relação ao apoio social pesquisas têm indicado que o apoio social em conjunto da escola (professores e outros atores do contexto educativo) e da família favorecem o desenvolvimento tanto das habilidades sociais quanto de competências acadêmicas (Marturano \& Pizato, 2015; Wang \& Holcombe, 2010). Tal apoio social auxilia os estudantes nos momentos de transição escolar, minimizando o estresse e promovendo a adaptação do aluno ao novo contexto (Langenkamp, 2010).

Não existe na literatura um consenso a respeito de aspectos terminológicos, operacionais e teóricos sobre o apoio social (Gonçalves, Pawlowski, Bandeira, \& Piccinini, 2011). No presente estudo, adotou-se o conceito de percepção de apoio social definido por Vaux et al. (1986) como a forma como a pessoa se percebe acolhida e apoiada por outros significativos, tais como membros da família, colegas e professores e que influenciam os seus comportamentos, pensamentos e sentimentos. A percepção de apoio social influencia positivamente o bem-estar físico e psicológico, autoestima, autoconceito e a motivação para o sucesso em vários setores da vida durante a adolescência, como, por exemplo, o desempenho escolar (Dias et al., 2015; Glozah \& Pevalin, 2014; Mackinnon, 2012).

$\mathrm{O}$ apoio social recebido e percebido pela pessoa modifica-se constantemente ao longo do ciclo vital (Gonçalves et al., 2011). Contudo, na adolescência a possibilidade de participação em múltiplos contextos e a maior autonomia do adolescente torna relevante identificar as características das diferentes fontes de apoio social (Squassoni \& Matsukura, 2014). Nessa direção, pesquisas (Antunes \& Fontaine, 2008; Olsson, Hagekull, Giannotta, \& Ahalander, 2016) indicam três fontes de apoio importantes: a família, os amigos e as pessoas da escola (professores). 
$\mathrm{Na}$ adolescência, apesar de a maior proximidade com pares, estudos encontraram que os membros familiares não deixam de ser referência de segurança para os adolescentes, caracterizando-se como apoio social complementar ao percebido pelos amigos (Alves \& Dell'Aglio, 2015a; Amparo, Galvão, Alves, Brasil, \& Koller, 2008; Olsson et al., 2016; Squassoni \& Matsukura, 2014). A percepção de apoio social da família possibilita que os adolescentes se sintam seguros e estabeleçam contatos em outros contextos, levando ao aumento da autoestima, do bem-estar, de sentimentos de aceitação facilitando a integração com pares (Olsson et al., 2016; Tomé \& Matos, 2012).

No contexto escolar, o apoio social dos amigos pode exercer influência positiva no desempenho acadêmico pelo fato dos adolescentes se perceberem num grupo que se identificam e que podem recorrer frente dificuldades não atendidas pelos professores (Dias et al., 2015; Tomé \& Matos, 2012). Assim, os amigos seriam uma fonte de aprendizagem de competências escolares e habilidades sociais, servindo de sustenção social e podendo exercer uma influência positiva na opção de um estilo de vida mais saudável (Del Prette \& Del Prette, 2009; Wang \& Holcombe, 2010). Porém, o relacionamento com pares também têm sido associado a comportamentos de risco na adolecência, tais como abuso de substâncias ilicítas e deliquência (Alves \& Dell'Aglio, 2015b; Olsson et al., 2016).

A escola pode ser um ambiente que possibilita aos adolescentes não apenas conteúdos acadêmicos, mas também pode ser um lugar previsível e com regras claras que o ajudam a sentir protegido (Bokhorst, Sumter, \& Westenberg, 2010; Dias et al., 2015; Machado, Yunes, \& Silva, 2014). Assim, evidências científicas têm demonstrado que a percepção do apoio social dos professores é associada com melhores níveis de bem-estar, autoestima e rendimento acadêmico, assim como menor chance de desenvolver problemas de comportamento (Dotterer \& Lowe, 2011; Glozah \& Pevalin, 2014; Tian, Zhao, \& Huebner, 2015). Nessa direção, pesquisas sugerem que há uma influência positiva do contexto escolar no desempenho acadêmico, pois os alunos sentem-se mais motivados e interessados nas atividades escolares quando os contextos de sala de aula favoreciam suas necessidades de relacionamento interpessoal (Amparo et al., 2008; Dias et al., 2015; Dotterer \& Lowe, 2011).

Portanto, quando os contextos escolares, familiares e as relações de amizade oferecem mais apoio social verifica-se um melhor desenvolvimento socioemocional dos alunos com baixo desempenho escolar, dando-lhes melhores condições de persistirem nos estudos sem que haja abandono (Hymel \& Ford, 2012). Inversamente, notas baixas dos alunos associadas ao apoio familiar limitado, a falta de conexão emocional do estudante com a escola e com os professores e o relacionamento precário com os colegas seriam fatores de maior influência para evasão escolar (Dias et al., 2015; Langenkamp, 2010).

O baixo desempenho escolar pode estar associado com vários fatores presentes nos contextos escolar e familiar dos estudantes e influenciado por aspectos das políticas públicas educacionais (França \& Gonçalves, 2003; Wang $\&$ Holcombe, 2010). No Brasil, cerca de 39\% dos alunos abandonam a escola antes de concluir o E.F., especialmente aqueles com histórias de reprovações (Oliveira \& Saraiva, 2015). Alguns estudos encontraram relação entre o apoio social da família, professores e amigos e o sucesso acadêmico, mostrando que o contexto social influencia o desempenho escolar (Glozah \& Pevalin, 2014; Mackinnon, 2012; Nunes et al., 2014). Com isso, quanto mais suporte social os alunos tiveram mais facilmente os mesmos poderão enfrentar os desafios do percurso escolar (Machado et al., 2014).

Apesar de tais evidências, a revisão de literatura demonstrou que no contexto nacional não há pesquisas que procurassem investigar o impacto da percepção de apoio social e das habilidades sociais dos alunos sobre o desempenho escolar durante nos últimos anos do Ensino Fundamental. Foi possível identificar uma lacuna no conhecimento nesse momento do ciclo escolar, que, como verificado anteriormente, apresenta número expressivo de evasão escolar. Nesse 
âmbito o presente estudo teve por objetivo testar um modelo de predição para o desempenho escolar, tendo como variáveis independentes as habilidades sociais, a percepção de apoio social da família, professores e pares e a reprovação dos estudantes. Assim, com as informações da presente pesquisa espera-se poder planejar programas de intervenção com os alunos, seus familiares e seus professores de modo a ampliar seus recursos interpessoais.

\section{Método}

\section{Participantes}

Foi realizado um estudo correlacional preditivo com uma amostra selecionada por conveniência. Participaram da pesquisa 311 alunos, de ambos os sexos, com idade média de 14,03 anos $(D P=1,23)$, que frequentavam os dois últimos anos do Ensino Fundamental (176 alunos do $8^{\circ}$ ano e 142 do $9^{\circ}$ ano) de três escolas públicas do Estado do Rio de Janeiro. As escolas apresentaram avaliação semelhante no Ideb, correspondente ao ano de 2013: escola $1=4,1$; escola $2=$ 4,$0 ;$ escola $3=4,0$.

\section{Instrumentos}

Inventário de Habilidades Sociais para Adolescentes - IHSA-Del-Prette (Del Prette \& Del Prette, 2009). Avalia as habilidades sociais de adolescentes a partir dos seus autorrelatos sobre situações cotidianas. Apresenta 38 itens que contemplam diferentes habilidades sociais divididas em seis fatores: (1) Empatia ("Ao notar que um(a) colega está triste ou com alguma dificuldade na escola, oferece apoio ou ajuda"); (2) Autocontrole ("Ao ser criticado pelos pais e professores consegue acalmar-se e controlar a irritação"); (3) Civilidade ("Quando alguém faz algo bom consegue elogiar e agradece quando recebe elogio"); (4) Assertividade ("Se acha errado fazer alguma coisa, mesmo os colegas pressionado, não faz o que os colegas querem"); (5) Abordagem Afetiva ("Quando quer fazer amizade, convida a pessoa para algum programa ou atividade"); (6) Desenvoltura Social ("Na escola consegue fazer apresentações orais em grupo quando solicitado"). As respostas estão dispostas em uma escala do tipo Likert de cinco pontos que varia de 0 (nunca) a 4 (sempre). O instrumento apresentou os seguintes índices de consistência interna para a amostra do estudo (Empatia $\alpha=0,81$; Autocontrole $\alpha=0,77$; Civilidade $\alpha=$ 0,83 ; Assertividade $\alpha=0,71$; Abordagem afetiva $\alpha=0,61$; Desenvoltura social $\alpha=0,57$; total do instrumento $\alpha=0,91$ ).

Escala de Percepção de Apoio Social (EPAS). É um instrumento desenvolvido por Vaux et al. (1986) para o contexto americano para examinar a percepção de apoio social de crianças e adolescentes em relação à família e aos amigos (Social Support Appraisal - SSA). A escala foi validada para a população de adolescentes brasileiros por Squassoni e Matsukura (2014). É composta por 30 itens disposto numa escala tipo Likert de 1 (Discordo totalmente) a 6 (Concordo totalmente) que contemplam quatro fatores: (1) Percepção de apoio social da família; (2) Percepção de apoio social dos amigos; (3) Percepção de apoio social dos professores; (4) e Percepção de apoio social dos outros em geral. Esse último fator não foi usado no presente estudo porque objetiva-se investigar o apoio social específico. $\mathrm{O}$ instrumento apresentou para a presente amostra os seguintes índices de consistência interna (família $\alpha=0,78$; amigo $\alpha$ $=0,74$; professor $\alpha=0,69$; total da escala $\alpha=$ 0,81 ).

Questionário de Informações Demográficas e Nivel Socioeconômico. Foi elaborado para este estudo para investigar informações sociais e demográficas, divididos em três partes: (a) informações gerais de aplicação; (b) informações sobre o participantes (nome, idade, sexo, cor e reprovação escolar - se já tinha sido reprovado de ano e quantas vezes); (c) informações sobre a família (número e indicação de quem residia com os alunos). Foi também empregado o Questionário de Classificação Econômica Brasil (Associação Brasileira de Empresas de Pesquisa [ABEP], 2011) que avalia o nível socioeconômico e permite a estratificação das famílias, em ordem decrescente de poder aquisitivo, em cinco classes: A (A1 e A2), B (B1 e B2), C, D e E. 


\section{Procedimentos}

Coleta de Dados. Após a entrega do Termo de Consentimento Livre e Esclarecido (TCLE), assinado pelos responsáveis legais dos alunos, a coleta de dados foi realizada, de forma coletiva nas salas de aula, no horário que foi combinado previamente com os professores. No início de cada aplicação (duração média 50 minutos) foi apresentado aos alunos o objetivo da pesquisa, a garantia de confidencialidade dos dados coletados e reiterado o caráter voluntário da sua participação. O projeto, condizente com Resolução 196/96 do Conselho Nacional de Saúde, foi aprovado pelo Comitê de Ética em Pesquisa da Universidade onde se realizou o estudo.

Análise dos Dados. A análise de dados foi executada com o software Statistical Package for the Social Sciences for Windows (SPSS, versão 18.0). Primeiramente, realizou-se a exploração das diferenças relacionadas ao sexo dos alunos (teste do Qui-quadrado) e análises descritivas (frequências e porcentagens) da variável reprovação escolar. Em seguida, cálculou-se a correlação entre as variáveis do estudo (coeficiente $r$ de Pearson). Por fim, foram testados e confirmados os pressupostos de normalidade, independência e multicolonearidade (Marôco, 2011) e, em seguida, procedeu-se a análise de regressão linear múltipla (método stepwise). $\mathrm{O}$ desempenho escolar foi a variável dependente e refere-se a média final (varia de 0,0 a 10,0), obtida pela soma das notas de todas as disciplinas computadas nas Atas de Resultados Finais do primeiro semestre do ano em que a coleta foi realizada. Foram consideradas como variáveis independentes: reprovação escolar; o total do IHSA-Del-Prette; os três fatores da EPAS (percepção de apoio social dos amigos, percepção de apoio social da família e percepção de apoio social dos professores).

\section{Resultados}

\section{Informações Demográficas}

Houve uma distribuição equilibrada em relação ao sexo dos participantes (173 meninas e 145 meninos $-\chi 2=2,46, p=0,11)$. No que se refere a cor declarada pelos alunos encontrou- -se que $43,08 \%$ da amostra informaram ser pardos, $29,87 \%$ brancos, $20,44 \%$ negros, $4,09 \%$ amarelos, 2,52\% indígenas. No que diz respeito ao nível socioeconômico das famílias, $37,10 \%$ pertenciam à classe $\mathrm{B} 2,28,90 \%$ à classe $\mathrm{C} 1$, $20,40 \%$ à classe $\mathrm{B} 1,8,50 \%$ à classe $\mathrm{C} 2,4,40 \%$ à classe $\mathrm{A} 2,0,30 \%$ à classe $\mathrm{D}$ e 0,30 à classe $\mathrm{A} 1$.

Em relação a experiência com a reprovação escolar, 200 alunos $(64,3 \%)$ informaram nunca ter tido reprovação, enquanto $111(35,7 \%)$ indicaram ter reprovado de ano pelo menos uma vez $(n=68), 38$ alunos duas vezes e cinco alunos mais de três vezes. Os dados mostraram que os adolescentes do sexo masculino $(n=60)$ reprovaram mais que as meninas $(n=50)$, porém o teste Qui-quadrado não indicou diferença significativa em relação ao sexo $(\chi 2=0,73, p=0,39)$. Os alunos que informaram ter reprovado de ano tinham idade entre 15 e 17 anos.

\section{Análises de Correlação e Regressão}

Os resultados indicaram correlação negativa moderada entre a reprovação e o desempenho escolar dos alunos $(r=-0,41 ; p=0,001)$. Com exceção da percepção de apoio social dos amigos, foram encontradas correlações positivas fracas e moderadas entre o total das habilidades sociais dos alunos $(r=0,26 ; p=0,001$, percepção de apoio social da família $(r=0,18 ; p=0,002)$ e dos professores $(r=0,22 ; p=0,001)$ e o desempenho escolar dos estudantes.

A Tabela 1 apresenta as variáveis preditoras para o desempenho escolar dos alunos. O modelo significativo $[F(3,30)=29,44, p=0,01]$ apresentado na Tabela 1 mostrou que a reprovação escolar, o total das habilidades sociais e a percepção de apoio social dos professores explicaram $22 \%$ da variabilidade do desempenho escolar dos alunos. Os resultados indicaram que a reprovação do aluno teve maior peso sobre o desempenho escolar. $\mathrm{O}$ valor do coeficiente estandardizado $(\beta)$ negativo indicou uma associação negativa entre a reprovação e o desempenho escolar. As habilidades sociais dos alunos e sua percepção de apoio social do professor correlacionaram-se positivamente com o desempenho escolar dos alunos. 
Tabela 1

Modelo de Regressão para a Predição do Desempenho Escolar dos Alunos ao Final do Ensino Fundamental

\begin{tabular}{ccccccc}
\hline Preditores & $M(D P)$ & Const. & $B($ EP $B)$ & $\beta$ & $95 \%$ IC & $R^{2}$ \\
\hline Reprovação escolar & $0,36(0,48)$ & $6,14^{*}$ & $-1,00(0,14)$ & $-0,36^{*}$ & {$[-1,28 ;-0,72]$} & $0,16^{*}$ \\
Habilidades Sociais & $90,68(24,39)$ & $5,13^{*}$ & $0,01(0,01)$ & $0,16^{*}$ & {$[0,03 ; 0,01]$} & $0,20^{*}$ \\
Apoio Social do Professor & $27,23(6,75)$ & $4,74^{*}$ & $0,02(0,01)$ & $0,13^{*}$ & {$[0,01 ; 0,04]$} & $0,21^{*}$ \\
\hline
\end{tabular}

Nota. $N=311 . B=$ coeficiente de regressão não padronizado, EP $B=$ Erro Padrão do coeficiente de regressão não padronizado; $\beta=$ coeficiente de regressão padronizado; IC = Intervalo de Confiança; $R^{2}=$ coeficiente de determinação ajustado. $* p<0,01$.

\section{Discussão}

Os últimos anos do Ensino Fundamental (E.F) são um momento do ciclo escolar crítico na trajetória acadêmica porque os alunos enfrentam diversas dificuldades que envolvem fatores socioeconômicos, políticos e interpessoais que levam ao baixo desempenho escolar e, consequentemente, aumentam a chance de abandono e evasão (Inep, 2015). Contudo, os anos finais do E.F. tem recebido pouca atenção das pesquisas. Assim, o presente estudo teve por objetivo investigar o impacto de variáveis dos alunos (habilidades sociais dos alunos e vivências de reprovações) e do contexto (percepção de apoio social) sobre o desempenho escolar nos dois últimos anos do E.F.

Os resultados indicaram que $35 \%$ da amostra já tinha reprovado de ano pelo menos uma vez, sem diferença entre os sexos. Embora $\mathrm{Nu}-$ nes et al. (2014) também não tenha encontrado diferenças entre meninos e meninas, as autoras identificaram que cerca de $50 \%$ dos participantes do estudo já tinham sofrido reprovação escolar. A pesquisa de Ortigão e Aguiar (2013) também evidenciou que até o $5^{\circ}$ ano do E.F., $40 \%$ das meninas já tinham reprovado de ano, enquanto para os meninos esse percentual era quase de $60 \%$.

Os dados da pesquisa mostraram que os alunos que haviam reprovado de ano tinham idade entre 15 e 17 anos, idade esta compatível para estar no Ensino Médio. No estudo realizado por Souza et al. (2012) os autores encontraram que a evasão escolar no decorrer do ano letivo é maior entre os alunos que apresentam idade incompa- tível a série estudada. Ou seja, os alunos com histórico de atraso escolar manifestam maior tendência de abandonar a escola durante o ano letivo, do que de um ano para o outro. Nunes et al. (2014) também identificou que a reprovação escolar associou negativamente com as expectativas dos estudantes de concluir o Ensino Médio e entrar na Universidade.

Isso chama a atenção para a possibilidade de os alunos entrarem num ciclo de fracasso escolar que prejudica tanto a qualidade da permanência e a conclusão do E.F., quanto o ingresso no E.M. (Alves et al., 2007; Bandeira et al., 2006; Jacomini, 2009). Deve-se ressaltar que fracasso ou o sucesso no processo de aprendizagem escolar é em menor frequência determinado por questões individuais do que por mecanismos interativos, pedagógicos, institucionais e políticos (Dazzani et al., 2014). Assim, o foco do olhar a respeito do baixo desempenho escolar não deve ser baseado em dificuldades psicológicas ou maturacionais dos alunos; mas sim na investigação dos processos relacionais entre os alunos e os contextos familiares, escolares e institucionais (Marturano \& Elias, 2016).

Os resultados das análises de correlação vão ao encontro da literatura, indicando que quanto mais reprovação, menor era o desempenho escolar dos estudantes ao final do E.F. (Womack et al., 2011). De forma semelhante a outros estudos recursos dos alunos como as habilidades sociais (Cia \& Costa, 2012; Feitosa et al., 2012; Lopes et al., 2013; Hall \& DiPerna, 2016; Marturano \& Pizato, 2015; Milson \& Glanville, 2010) e a percepção de apoio social da família e dos professores (Dias et al., 2015; Glozah \& Pevalin, 2014: 
Mackinnon, 2012) se associaram positivamente com o desempenho escolar dos estudantes. No contexto da transição escolar do E.F. para o E.M., o apoio social da família, dos amigos e dos professores levam a bons resultados acadêmicos para os alunos (Langenkamp, 2010). Marturano e Pizato (2015) apontaram para a importância das redes de apoio e que estas associadas aos recursos do aluno (habilidades sociais) amenizam os impactos negativos que esta transição pode ocasionar.

Os resultados do modelo de regressão testado indicaram que o desempenho escolar ao final do E.F. foi predito, em ordem de influência, pela reprovação escolar, habilidades sociais e percepção de apoio social dos professores, explicando em seu conjunto $22 \%$ da variabilidade do desempenho. A reprovação escolar foi a variável com maior peso no desempenho escolar dos alunos, apresentando uma associação negativa com o mesmo. Outras pesquisas encontraram resultados semelhantes, sinalizando que a reprovação escolar é um fator de risco para o desenvolvimento socioemocional e acadêmico dos estudantes, levando a distorção idade-série e abandono escolar (Alves et al., 2007; Bandeira et al., 2006; Jacomini, 2009).

Conforme Ortigão e Aguiar (2013), isso ocorre porque a reprovação escolar prejudica as atividades acadêmicas e as relações interpessoais dos alunos e estes tendem a ficar desmotivados e, consequentemente, abandonarem os estudos. Muitas vezes os próprios professores e colegas estigmatizam e discriminam os alunos repetentes (Faissol \& Bastos, 2014). Assim, esses estudantes acabam por se isolar (ou serem isolados), não participam das atividades propostas e não apresentam interesse em realizar as proposições referentes à aprendizagem (Almeida \& Sartori, 2012).

De acordo com a literatura prévia, as habilidades sociais dos estudantes predisseram positivamente o desempenho escolar. Vários estudos mostraram que um bom repertório de habilidades sociais auxilia a competência acadêmica e o bom desempenho escolar, pois as habilidades sociais são recursos do indivíduo que ajudam o processo de aprendizagem (Caemmerer \& Keith, 2015; Hall \& DiPerna, 2016; Zambon \& Rose, 2012). Relatar os sentimentos, pedir ajuda, dar e receber apoio são capacidades que podem ajudar o aluno a expressar seus sentimentos frente ao baixo desempenho escolar e até pedir ajuda aos amigos, pais e professores para sair desta situação vulnerável (Lopes et al., 2013; Zambon \& Rose, 2012; Womack et al., 2011). Portanto, as habilidades sociais estimulam a capacidade de tomada de decisão e a diminuição de comportamentos disfuncionais e de risco, auxiliando o desempenho escolar (Feitosa et al., 2012).

Dentre os três tipos de apoio social testado no modelo de regressão, apenas a percepção de apoio social dos professores foi um preditor positivo do desempenho escolar dos alunos. Embora na adolescência, as relações interpessoais se diversifiquem, pesquisas encontraram que os adolescentes percebem mais o apoio social da família do que pares e professores (Alves \& Del'Aglio, 2015a; Amparo et al., 2008; Olsson et al., 2016; Squassoni \& Matsukura, 2014). Assim, pode-se supor que ainda que os professores sejam percebidos como pouca fonte de apoio social, a sua influência no desempenho escolar é importante e precisa ser incentivada (Alves \& Dell'Aglio, 2015a; Bolsoni-Silva et al., 2013; Dias et al., 2015), principalmente na transição do E.F. para o E. M. Nesse sentido, Berry e O Connor (2010) ao realizarem um estudo longitudinal com 1364 crianças do maternal até o $6^{\circ}$ ano do E.F., encontraram uma correlação positiva entre a qualidade da relação professor-aluno e as habilidades sociais dos estudantes com problemas internalizantes.

Embora a percepção de apoio social da família e dos amigos não tenha entrado no modelo de regressão os mesmos devem ser estimulados. A percepção de apoio social da família ajuda o aluno a enfrentar os desafios e a sentir-se mais acolhido e mais protegido (Squassoni \& Matsukura, 2014). Já a comunicação afetiva da família auxilia o aluno na socialização, na ampliação da rede de contato e protege de situações de risco como as amizades que contribuem para comportamentos disfuncionais (Alves \& 
Dell'Aglio, 2015b; Olsson et al., 2016). Os adolescentes que têm mais contato e um bom relacionamento com os pais mostram-se mais felizes e mais satisfeitos com a vida (Tomé \& Matos, 2012). Assim, a percepção de apoio social da família associada a uma relação recíproca, com boa comunicação e um relacionamento positivo contribui para o aluno ter mais comportamentos saudáveis e um bom desempenho escolar (França \& Gonçalves, 2003; Glozah \& Pavalin, 2014).

Da mesma maneira, as amizades podem auxiliar o aluno que possui baixo desempenho escolar, por exemplo, a tirar dúvida com outros colegas além de precisar recorrer ao professor. Além do mais, estes possuem a mesma linguagem e estão na mesma etapa de desenvolvimento (no caso a adolescência), o que pode favorecer a troca de experiências (Tomé \& Matos, 2012). Assim, pode-se sugerir que as relações de amizade recíprocas contribuem para bons resultados escolares dos alunos em seu cotidiano (Wang \& Holcombe, 2010), ainda que algumas relações de amizade afetem negativamente o desenvolvimento dos estudantes, por exemplo, uso de drogas, violência e comportamentos agressivos (Alves \& Dell'Aglio, 2015b). Por isso, todas as redes de apoio são importantes para auxiliar o desenvolvimento, pois se uma não oferece suporte, a outra pode oferecer amparo para que o sujeito se sinta acolhido. Sendo assim, não basta o aluno se sentir apoiado, mas também receber recursos materiais, sociais e afetivos das suas redes de apoio para promover o seu desenvolvimento saudável (Machado et al., 2014).

\section{Considerações Finais}

Em linhas gerais, os resultados indicaram que as habilidades sociais (recursos do aluno) e a percepção de apoio social do professor (recursos do contexto escolar) impactam positivamente no desempenho escolar dos alunos, podendo auxilia-los ao longo da sua trajetória escolar e, principalmente, nos momentos de transição como no final do Ensino Fundamental. Algumas limitações do presente estudo devem ser consideradas.
Em primeiro lugar, os resultados são específicos de uma determinada amostra que foi selecionada por conveniência a partir de algumas escolas públicas e privadas situadas no Estado do Rio de Janeiro.

Em segundo lugar, os dados também foram coletados a partir das percepções dos alunos e, desse modo, seria interessante que futuros estudos incluíssem informações coletadas com outros informantes, como pais e professores. Em terceiro lugar, o valor do coeficiente de regressão encontrado é considerado pequeno, o que sinaliza que futuros estudos podem incluir outras variáveis, tais como clima escolar, práticas educativas parentais e percepção de estressores escolares. Por fim, deve-se considerar que o desempenho escolar foi mensurado por meio das médias escolares que são medidas não padronizadas de competências. Desse modo, se, por um lado, tais medidas oferecem dados contextualizados da realidade educacional das escolas pesquisas, por outro, limitam a comparação com outros locais. Assim, outros instrumentos de medida de desempenho poderiam ser utilizados em futuros estudos como a Prova Brasil ou o Teste de Desempenho Escolar, ou mesmo medidas de competência acadêmica, como o Sistema de Avaliação de Habilidades Sociais (SSRS) na versão professores. Por fim, as análises estatísticas não permitem conclusões definitivas nem estabelecer relações de causa e efeito, mas apenas inferências correlacionais.

A trajetória escolar é um fenômeno multifatorial que é influenciado por variáveis individuais, contextuais (família, escola e comunidade), sociais, econômicas e políticas. Deve-se haver investimento em políticas públicas educacionais, principalmente nos últimos anos do Ensino Fundamental para melhorar a qualidade do ensino, assim como as condições estruturais e salariais do trabalho docente. O estudo contribuiu para evidenciar algumas variáveis que podem ser focalizadas em futuros programas de intervenção com alunos, família e agentes educativos. Tais programas de intervenção podem auxiliar os alunos abaixo da média escolar e que tiveram alguma reprovação e, assim, mantê-los na escola para concluir os estudos. 


\section{Referências}

Almeida, T. F. S., \& Sartori, J. (2012). A relação entre desmotivação e o processo de ensino-aprendizagem. Revista Monografias Ambientais, 8(8), 1870-1886. Recuperado em http://cascavel. ufsm.br/revistas/ojs-2.2.2/index.php/remoa/in$\operatorname{dex}$

Alves, A. M. L. (2013). Os ciclos: A experiência da organização do ensino no Rio de Janeiro e Niterói. Educação e Foco, 17(3), 87-115. Recuperado em http://www.ufjf.br/revistaedufoco/ files/2013/10/cap-04.pdf

Alves, C. F., \& Dell'Aglio, D. D. (2015a). Percepção de apoio social de adolescentes de escolas públicas. Revista de Psicologia da IMED, 7(2), 89-98. doi:10.18256/2175-5027/psico-imed. v7n2p89-98

Alves, C. F., \& Dell'Aglio, D. D. (2015b). Apoio social e comportamentos de risco na adolescência. Psico, 46(2), 165-175. doi:http://dx.doi. org/10.15448/1980-8623.2015.2.18250

Alves, F., Ortigão, I., \& Franco, C. (2007). Origem social e risco de repetência: Interação entre raça-capital econômico. Cadernos de Pesquisa, 37(130), 161-180. doi:10.1590/S010015742007000100008

Amparo, D. M., Galvão, A. C. T., Alves, P. B., Brasil, K. T., \& Koller, S. H. (2008). Adolescente e jovens em situação de risco psicossocial: Redes de apoio social e fatores pessoais de proteção. Estudos de Psicologia (Natal), 13(2), 165-174. doi:10.1590/S1413-294X2008000200009

Antunes, C., \& Fontaine, A. M. (2008). Percepção de apoio social na adolescência: Análise fatorial confirmatória da escola Social Support Appraisails. Paideia (Ribeirão Preto), 15(32), 355-366. Recuperado em http://sites.ffclrp.usp.br/paideia/ artigos/32/04.htm

Associação Brasileira de Empresas de Pesquisa. (2011). Critério de classificação econômica Brasil. Recuperado em http://www.abep.org/ criterio-brasil

Bandeira, M., Rocha, S. S., Pires, L. G., Del Prette, Z. A. P., \& Del Prette, A. (2006). Competência acadêmica de crianças do Ensino Fundamental: Características sociodemográficas e relação com habilidades sociais. Interação em Psicologia, 10(1), 53-62. Recuperado em http://ojs.c3sl.
ufpr.br/ojs/index.php/psicologia/article/viewFile/5773/4209

Beauchamp, M. H., \& Anderson, V. (2010). Social: An integrative framework for the development of social skills. Psychological Bulletin, 136(1), 39-64. doi:10.1037/a0017768

Berry, D., \& O`Connor, E. (2010). Behavioral risk, teacher-child relationships, and social skill development across middle childhood: A child-byenvironment analysis of change. Journal of Applied Developmental Psychology, 31(1), 1-14. doi:10.1016/j.appdev.2009.05.001

Bokhorst, C. L., Sumter, S. R., \& Westenberg, P. M. (2010). Social support from parents, friends, classmates, and teachers in children and adolescents aged 9 to 18 years: Who is perceived as most supportive? Social Development, 19(2), 417426. doi:10.1111/j.1467-9507.2009.00540.x

Bolsoni-Silva, A. T., Verdu, A. C. M. A., Carrara, K., Melchiori, L. E., Leite, L. P., \& Calais, S. L. (2013). Ampliando comportamentos pró-éticos dos alunos: Relato de pesquisa e intervenção com educadores do Ensino Fundamental. Temas em Psicologia, 21(2), 347-359. doi:10.9788/ TP2013.2-04

Caemmerer, J. M., \& Keith, T. Z. (2015). Longitudinal, reciprocal effects of social skills and achievement from kindergarten to eighth grade. Journal of School Psychology, 53, 265-281. doi:10.1016/j.jsp.2015.05.001

Cia, F., \& Costa, C. L. S. (2012). Desempenho acadêmico nas séries do ensino fundamental: Relação com o desenvolvimento social. Psicologia e Argumento, 30(68), 109-118. Recuperado em http://www2.pucpr.br/reol/pb/index.php/ pa?dd1 $=5889 \& d d 99=$ view $\& d d 98=$ pb

D'Abreu, L. C. F., \& Marturano, E. M. (2010). Associação entre comportamentos externalizantes e baixo desempenho escolar: Uma revisão de estudos prospectivos e longitudinais. Estudos de Psicologia (Natal), 15(1), 43-51. doi:http://dx.doi. org/10.1590/S1413-294X2010000100006

Dazzani, M. V. M., Cunha, E. de O., Luttigards, P. M., Zucoloto, P. C. S. V., \& Santos G. L. (2014). Queixa escolar: Uma revisão crítica da produção científica nacional. Revista Quadrimestral da Associação Brasileira de Psicologia Escolar e Educacional, 18(3), 421-428. doi:10.1590/2175$3539 / 2014 / 0183762$ 
Del Prette, Z. A. P., \& Del Prette, A. (2009). Inventário de Habilidades Sociais para adolescentes (IHSA-DEL-PRETTE): Manual de aplicação, apuração e interpretação. São Paulo, SP: Casa do Psicólogo.

Del Prette, Z. A. P., \& Del Prette, A. (2010). Habilidades sociais e análise do comportamento: Proximidade histórica e atualidades. Revista Perspectivas, 1(2), 104-115. Recuperado em http://porteiras.r.unipampa.edu.br/portais/ cap/files/2013/12/TREINAM-HABLDD-SOCIAIS-104-115_RP_2010_01_02.pdf

Dias, A., Oliveira, J. T., Moreira, P. A. S., \& Rocha, L. (2015). Percepção dos alunos acerca das estratégias de promoção do sucesso educativo e envolvimento com a escola. Estudos de Psicologia (Campinas), 32(2), 187-199. doi:10.1590/0103$-166 \times 2015000200004$

Dotterer, A. M., \& Lowe, K. (2011). Classroom context, school engagement, and academic achievement in early adolescence. Journal Youth Adolescence, 40, 1649-1660. doi:10.1007/ s10964-011-9647-5

Elias, L. C. S., \& Marturano, E. M. (2014). "Eu posso resolver problemas" e oficinas de linguagem: Intervenções para queixa escolar. Psicologia: Teoria \& Pesquisa, 30(1), 35-44. doi:10.1590/ S0102-37722014000100005

Faissol, K., \& Bastos, M. C. (2014). Projeto Refazer: Uma reflexão da reprovação a partir do olhar do aluno. Revista de Psicologia da Criança e do Adolescente, 5(1), 201-210. Recuperado em http://revistas.lis.ulusiada.pt/index.php/rpca/article/view/1132

Feitosa, F. B., Del Prette, Z. A. P., \& Del Prette, A. (2012). Social skills and academic achievement: The mediating function of cognitive competence. Temas em Psicologia, 20(1), 61-70. Retrived from http://pepsic.bvsalud.org/pdf/tp/ v20n1/v20n1a06.pdf

França, M. T. A., \& Gonçalves, F. O. (2003). Sistemas públicos de ensino fundamental e a perpetuação da desigualdade: Democracia e qualidade educacional como promotoras de justiça social. Revista Brasileira de Estudos de População, 29(2), 303-322. Recuperado em www.scielo.br/ pdf/rbepop/v29n2/a06v29n2.pdf

Glozah, F. N., \& Pevalin, D. J. (2014). Social support, stress, health, and academic success in Ghanaian adolescents: A path analysis. Journal of Adolescence, 37, 451-460. doi:10.1016/j.adolescence.2014.03.010

Gonçalves, T. R., Pawlowski, J., Bandeira, D. R., \& Piccinini, C. A. (2011). Avaliação de apoio social em estudos brasileiros: Aspectos conceituais e instrumentos. Ciência \& Saúde Coletiva, 16(13), 1755-1769. doi:10.1590/S141381232011000300012

Hall, G. E., \& DiPerna, J.C. (2016). Childhood social skills as predictors of middle school academic adjustment. Journal of Early Adolescence, 3(4), 1-27. doi:10.1177/0272431615624566

Hymel, S., \& Ford, L. (2012). Conclusão escolar e sucesso acadêmico: O impacto da competência socioemocional precoce. In T. R. E. Tremblay, M. Boivin, \& M. P. RDeV (Eds.), Enciclopédia sobre o Desenvolvimento na Primeira Infância (pp. 1-8). Montreal: Centre D'excellence pour le Développement des Jeunes Enfants. Recuperado em http://www.enciclopedia-crianca.com/sites/ default/files/textes-experts/pt-pt/2612/conclusao-escolar-e-sucesso-academico-o-impacto-dacompetencia-sociomocional-precoce-.pdf

Índice de Desenvolvimento da Educação Básica. (2013). Brasília, DF: Ministério da Educação. Recuperado em http://ideb.inep.gov.br/resultado

Inglés, C. J., Martínez-González, A. E., García-Fernández, J. M., \& Ruiz-Esteban, C. (2011). Conducta prosocial y motivación académica en estudiantes españoles de Educación Secundaria Obligatoria. Universita Psychologica, 10(2), 451-465. Retrieved from http://revistas. javeriana.edu.co/index.php/revPsycho/article/ view/756

Ingul, M. J., Klockner, C. A., Silverman, W. K., \& Nordahl, H. M. (2012). Adolescent school absenteeism: Modelling social and individual risk factors. Child and Adolescent Mental Health, 17(2), 93-100. doi:10.1111/j.1475-3588.2011.00615

Instituto Nacional de Estudos e Pesquisas Educacionais Anísio Teixeira. (2015). Censo escolar 2015. Brasília, DF: Autor. Recuperado em http://portal.inep.gov.br/basica-censo

Jacomini, M. A. (2004). A escola e os educadores em tempo de ciclos e progressão continuada: Uma análise das experiências no estado de São Paulo. Educação e Pesquisa, 30(3), 401-418. Recuperado em www.scielo.br/pdf/ep/v30n3/ a02v30n3.pdf 
Jacomini, M. A. (2009). Educar sem reprovar: Desafio de uma escola para todos. Educação e Pesquisa, 35(3), 557-572. Recuperado em http:// www.scielo.br/pdf/ep/v35n3/10.pdf

Langenkamp, A. G. (2010). Academic vulnerability and resilience during the transition to high school: The role of social relationships and district context. Sociology of Education, 83(1), 1-19. doi:10.1177/0038040709356563

Lopes, D. C., Del Prette, Z. A. P., \& Del Prette, A. (2013). Recursos multimídia no ensino de habilidades sociais a crianças de baixo rendimento acadêmico. Psicologia: Reflexão $e$ Crítica, 26(3), 451-458. doi:10.1590/S010279722013000300004

Loureiro, C., Ferreira, M. M. F., \& Santos, M. R. (2013). Identificação dos fatores determinantes no desenvolvimento das competências sociais dos adolescentes. Revista de Enfermagem, 3(10), 79-88. doi:10.12707/RIII12114

Machado, J. A., Yunes, M. A M., \& Silva, G. F. (2014). A formação continuada de professores em serviço na perspectiva da abordagem ecológica do desenvolvimento humano. Revista Contrapontos, 14(3), 512-526. doi:10.14210/contrapontos.v14n3.p512-526

Mackinnon, S. P. (2012). Perceived social support and academic achievement: Cross-Lagged panel and bivariate growth curve analyses. Journal Youth Adolescence, 41(2), 474-485. doi:10.1007/ s10964-011-9691-1

Marôco, J. (2011). Analise estatística com o SPSS Statistics (5. ed.). Pero Pinheiro, Portugal: ReportNumber.

Marturano, E. M., \& Elias, L. C. S. (2016). Família, dificuldades no aprendizado e problemas de comportamento em escolares. Educar em Revista, 59, 123-139. doi:10.1590/0104-4060.44617

Marturano, E. M., \& Pizato, E. C. G. (2015). Preditores de desempenho escolar no $5^{\circ}$ ano do Ensino Fundamental. Psico, 46(1), 16-24 Recuperado em http://revistaseletronicas.pucrs.br/ojs/index. $\mathrm{php} /$ revistapsico/article/view/14850

Milson, A., \& Glanville, J. L. (2010). Factors mediating the relationship between social skills and academic grades in a sample of students diagnosed with learning disabilities or emotional disturbance. Remedial and Special Education, 31(4), 241-251. Retrieved from http://rse. sagepub.com/content/31/4/241
Moreira, J. O., Rosário, A. B., \& Santos, A. P. (2011). Juventude e adolescência: Considerações preliminares. Psico, 42(4), 457-464. Recuperado em http://revistaseletronicas.pucrs.br/ojs/index.php/ revistapsico/article/viewArticle/8943

Nunes, T. G., Pontes, F. A. R., Silva, L. I. C., \& Dell'Aglio, D. (2014). Fatores de risco e proteção na escola: Reprovação e expectativas de futuro de jovens paraenses. Revista Quadrimestral da Associação Brasileira de Psicologia Escolar e Educacional, 18(2), 203-210. doi:http://dx.doi. org/10.1590/2175-3539/2014/0182732

Oliveira, D. A., \& Saraiva, A. M. A. (2015). A relação entre educação e pobreza: A ascensão dos territórios educativos vulneráveis. Revista Educação Temática Digital, 17(3), 614-632. Recuperado em http://periodicos.sbu.unicamp.br/ojs/ index.php/etd/article/view/8638257/9849

Olsson, I., Hagekull, B., Giannotta, F., \& Ahalander, C. (2016). Adolescents and social support situations. Scandinavian Psychological Associations and John Wiley \& Sons, 31(1), 1-10. doi:10.1111/sjop.12282

Ortigão, M. I. R., \& Aguiar, G. S. (2013). Repetência escolar nos anos iniciais do Ensino Fundamental: Evidências a partir dos dados da Prova Brasil 2009. Revista Brasileira de Pedagogia, 94(237), 364-389. doi:10.1590/S217666812013000200003

Perrenoud, P. (2003). Sucesso na escola: Só o currículo, nada mais que o currículo. Cadernos de Pesquisa, 119, 9-27. Recuperado em http:// publicacoes.fcc.org.br/ojs/index.php/cp/article/ view/513/517

Souza, A. P., Ponczec, V. P., Oliva, B. T., \& Tavares, P. A. (2012). Fatores associados ao fluxo escolar no ingresso e ao longo do Ensino Médio no Brasil. Pesquisa e Planejamento Econômico, 42(1), 5-39. Recuperado em http://ppe.ipea.gov.br/index.php/ppe/article/viewFile/1322/1110

Squassoni, C. E., \& Matsukura, T. S. (2014). Adaptação transcultural da versão portuguesa do Social Support Appraisals para o Brasil. Psicologia: Reflexão e Crítica, 27(1), 71-80. doi:10.1590/ S0102-79722014000100009

Tian, L., Zhao, J., \& Huebner, E. S. (2015). Schoolrelated social support and subjective well-being in school among adolescents: The role of selfsystem factors. Journal of Adolescence, 45, 138148. doi:10.1016/j.adolescence.2015.09.003 
Tomé, G., \& Matos, M. G. (2012). Relação positiva com grupo de pares na adolescência. In M. G. de Matos \& G. Tomé (Eds.), Aventura Social: Promoção de competências e do capital social para um empreendedorismo com saúde na escola e na comunidade (pp. 111-125). São Paulo, SP: Moderna.

Vaux, A., Phillips, J., Holly, L., Thomson, B., Williams, D., \& Stewart, D. (1986). The Social Support Appraisals (SS-A) Scale: Studies of reliability and validity. American Journal of Community Psychology, 14(2), 195-219. doi:10.0910562/86/0400-0195505.00/0

Wang, M. T., \& Holcombe, R. (2010). Adolescents' perceptions of school environment, engagement, and academic achievement in middle school. American Educational Research Journal, 47(3), 633-662. doi:10.3102/0002831209361209
Womack, S. A., Marchant, M., \& Borders, D. (2011). Literature-based social skills Instruction: A strategy for students with learning disabilities. Intervention in School and Clinic, 46(3), 157-164. Retrieved from http://journals.sagepub.com/doi/ abs/10.1177/1053451210378164

Zambon, M. P., \& Rose, T. M. S. (2012). Motivação de alunos do ensino fundamental: Relações entre rendimento acadêmico, autoconceito, atribuições de causalidade e metas de realização. $E d u$ cação e Pesquisa, 38(4), 965-980. doi:10.1590/ S1517-97022012000400012

Recebido: 28/06/2016

$1^{a}$ revisão: $13 / 02 / 2017$

Aceite final: 28/02/2017

(C) O(s) autor(es), 2018. Acesso aberto. Este artigo está distribuído nos termos da Licença Internacional Creative Commons Atribuição 4.0 (http://creativecommons.org/licenses/by/4.0/), que permite o uso, distribuição e reprodução sem restrições em qualquer meio, desde que você dê crédito apropriado ao(s) autor(es) original(ais) e à fonte, fornecer um link para a licença Creative Commons e indicar se as alterações foram feitas. 